\title{
BMJ Open Protocol for process evaluation of CIVIC randomised controlled trial: Community- based InterVentions to prevent serIous Complications following spinal cord injury in Bangladesh
}

To cite: Hossain MS, Harvey LA, Liu $\mathrm{H}$, et al. Protocol for process evaluation of CIVIC randomised controlled trial: Community-based InterVentions to prevent serlous Complications following spinal cord injury in Bangladesh. BMJ Open 2018;8:e024226. doi:10.1136/ bmjopen-2018-024226

- Prepublication history and additional material for this paper are available online. To view these files, please visit the journal online (http://dx.doi. org/10.1136/bmjopen-2018024226).

Received 8 June 2018 Revised 14 June 2018 Accepted 25 June 2018

Check for updates

(C) Author(s) (or their employer(s)) 2018. Re-use permitted under CC BY-NC. No commercial re-use. See rights and permissions. Published by BMJ.

For numbered affiliations see end of article.

Correspondence to Professor Lisa A Harvey; lisa.harvey@sydney.edu.au

\section{ABSTRACT}

Introduction People with spinal cord injuries in lowincome and middle-income countries are highly vulnerable to life-threatening complications in the period immediately after discharge from hospital. We are conducting a randomised controlled trial in Bangladesh to determine whether all-cause mortality at 2 years can be reduced if health professionals regularly ring and visit participants in their homes following discharge. We will conduct a process evaluation alongside the trial to explain the trial results and determine the feasibility of scaling this intervention up in low-income and middle-income countries if it is found to be effective.

Methods and analysis Our process evaluation is based on the Realist and Reach, Effectiveness, Adoption, Implementation and Maintenance frameworks. We will use a mixed methods approach that uses both qualitative and quantitative data. For example, we will audit a sample of telephone interactions between intervention participants and the healthcare professionals, and we will conduct semistructured interviews with people reflective of various interest groups. Quantitative data will also be collected to determine the number and length of interactions between the healthcare professionals and participants, the types of issues identified during each interaction and the nature of the support and advice provided by the healthcare professionals. All quantitative and qualitative data will be analysed iteratively before the final analysis of the trial results. These data will then be triangulated with the final results of the primary outcome.

Ethics and dissemination Ethics approval was obtained from the institutional ethics committee at the site in Bangladesh and from the University of Sydney, Australia. The study will be conducted in compliance with all stipulations of its protocol, the conditions of ethics committee approval and the relevant regulatory bodies. The results of the trial will be disseminated through publications in peer-reviewed scientific journals and presentations at scientific conferences. Trial registration number ACTRN12615000630516.
Strengths and limitations of this study

- The process evaluation involves mixed methods and draws together data from many different sources to help explain the trial results and determine the feasibility of scaling this intervention up in low-income and middle-income countries.

- The Community-based InterVentions to prevent serlous Complications following spinal cord injury in Bangladesh (CIVIC) trial will be the first large randomised controlled trial to look at the effectiveness of any type of community-base support programme for people with spinal cord injuries in a low-income or middle-income country.

- The process evaluation relies on staff involved in the trial to collect some of the data. This may introduce bias.

- The process evaluation does not collect data from the early stages of the trial.

\section{INTRODUCTION}

There are no accurate data on the incidence of spinal cord injuries (SCI) in low-income countries such as Bangladesh but most working in the area believe that it could be as high as 70 per million. ${ }^{2}$ That is, three to four times that of high-income countries. ${ }^{3}$ Similarly, there are few accurate data about survival following SCI in these countries. ${ }^{4-7}$ However, our own estimates from one specialised service in Bangladesh indicate that $19 \%$ of people with SCI who are wheelchair-dependent and survive until discharge, die within 2 years. $^{8}$ Most are young males dying from complications such as sepsis due to pressure ulcers. $^{8-11}$ There is therefore a pressing need to find sustainable ways of supporting people 
with SCI in the community following discharge, particularly those at high risk of complications.

The Community-based InterVentions to prevent serIous Complications following spinal cord injury in Bangladesh (CIVIC) trial was designed to test the effectiveness of an inexpensive and sustainable model of community-based care that could be rolled out in Bangladesh and other low-income countries to support people with SCI following discharge. The model of care was developed over a number of years and over the course of a preliminary pilot study. ${ }^{12}$ It involves assigning healthcare professionals for 2 years to people with SCI as they are discharged from hospital. The healthcare professionals act like case managers and are in regular telephone contact with participants, and responsible for monitoring for complications, providing advice and support, and being a familiar point of contact for participants and their families. The assigned healthcare professionals also visit participants in their homes and provide participants with a small amount of financial assistance (\$AU80). The healthcare professionals are thus responsible for proactively supporting participants and their families on discharge and providing them with regular support and advice, as well as monitoring for early signs of complications.

The primary outcome of CIVIC trial is all-cause mortality at 2 years. Recruitment to the study commenced in July 2015 and finished in March 2018 with the final follow-up assessment due in March 2020. The process evaluation described in this paper will help explain the results of the trial and determine the feasibility of scaling up this intervention in Bangladesh and other low-income and middle-income countries if it is found to be effective.

\section{Aim}

The aims of the process evaluation are to:

1. Explain CIVIC trial results and specifically, to determine:

- Whether the intervention was delivered as intended.

- Whether the control was delivered as intended.

- The types of issues typically identified during each interaction between intervention participants and healthcare professionals.

- The nature of the support and advice provided by the healthcare professionals to the intervention participants.

- Participants' and healthcare professionals' perspectives on how, why and for whom the intervention did or did not work.

2. Determine the feasibility of scaling the intervention up in Bangladesh and other low-income and middle-income countries if it is found to be effective and specifically, to determine:

- The possible barriers and facilitators to scaling the intervention up in the future.

- Whether people with SCI would value the intervention.
- Whether healthcare service providers could employ and retain staff to provide the intervention.

- Whether the results are generalisable to other patients, healthcare service providers and countries.

\section{METHODS AND ANALYSES}

\section{Summary of CIVIC trial}

An investigator-initiated pragmatic randomised controlled trial is being undertaken. The trial was prospectively registered with the Australia New Zealand Clinical Trials Registry and the trial protocol has been published. ${ }^{13}$ In brief, 410 people with recent SCI who are wheelchair dependent and about to be discharged home from the Centre for the Rehabilitation of the Paralysed in Bangladesh are randomised to either an intervention or control group (see figure 1). Participants in the intervention group receive our model of community-based care for2 years in which they are assigned a healthcare professional who rings them every 2 weeks in the first year and every month in the second year, and visits them in their homes on three occasions over the 2 years. At each point of contact, the healthcare professional screens participants for early signs of complications, and provides them and their families with advice and support. In contrast, participants in the control group receive the care that is currently provided by the Centre for the Rehabilitation of the Paralysed. That is, at discharge participants are encouraged to ring the hospital if they develop any problems, and some are rung or visited on one occasion by hospital staff as part of the hospital's limited follow-up service.

The primary outcome is all-cause mortality at 2 years determined by blinded assessors interviewing next of kin (Bangladesh does not have a death registry). There are also a number of secondary outcomes including complications, depression, independence, quality of life and ability to participate in community activities.

The trial is powered to have a $80 \%$ probability of detecting an increase in survival from $83 \%^{8}$ to $93 \%$ at 2 years with a two-sided log-rank test, uniform follow-up time of 2 years, loss to follow-up in both groups of $15 \%$ at 2 years and an alpha of 0.05 . A trial-based economic evaluation will be conducted based on differences observed between groups in costs, overall survival and quality-adjusted survival at 2 years. This will enable an estimate of an incremental cost per quality adjusted life year of the intervention over standard care.

\section{The theoretical framework for our process evaluation}

Figure 2 outlines the framework of our process evaluation as recommended by the Medical Research Council's guidance on process evaluations of complex interventions. ${ }^{14}$ It provides a summary of the key questions and the proposed causal pathways between CIVIC trial intervention and outcomes within the context of the ultimate aim of the trial and intervention, namely to reduce complications and mortality in people with SCI after discharge from hospital. 


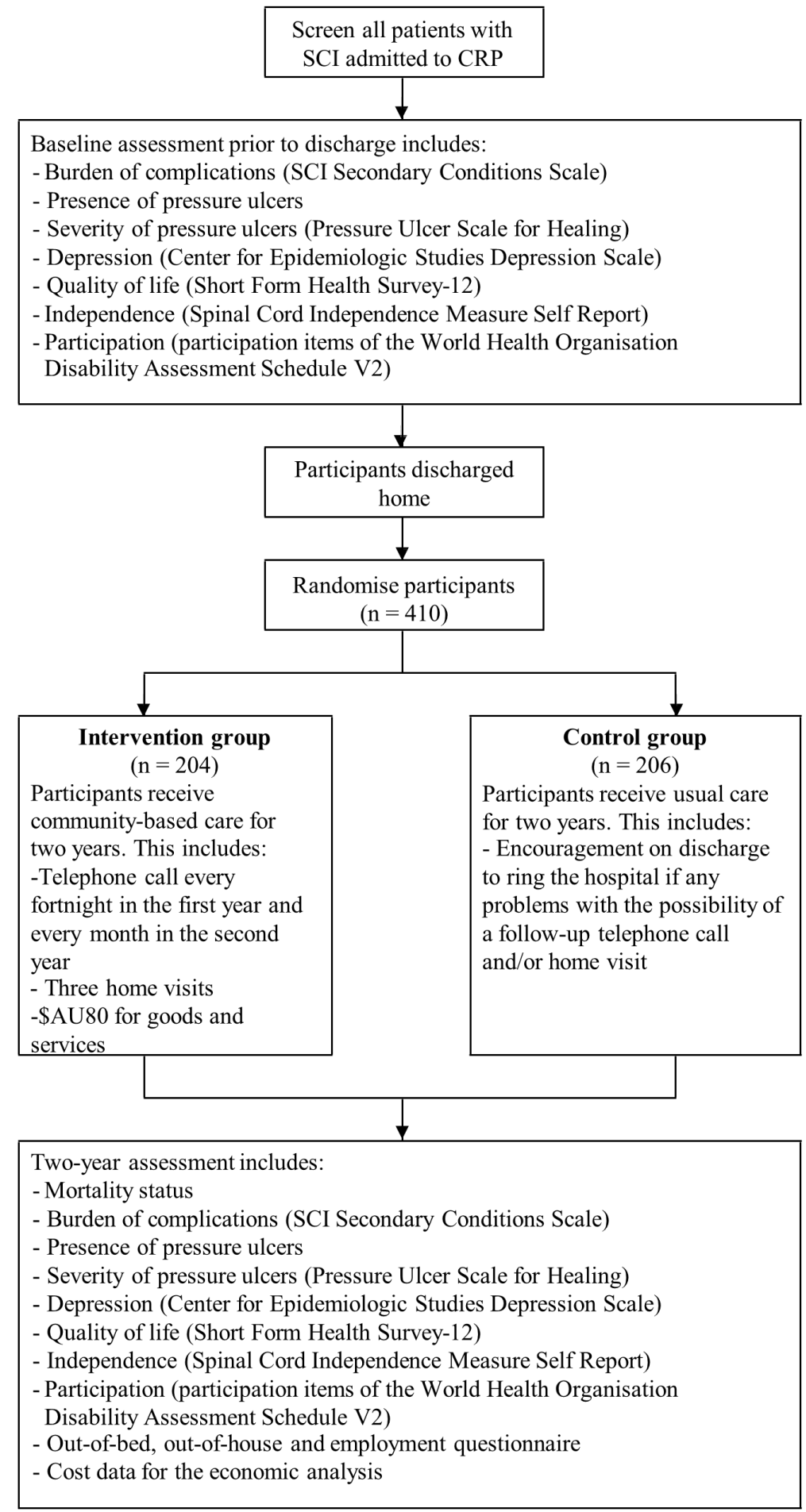

Figure 1 The CIVIC trial flow chart. CIVIC, Community-based InterVentions to prevent serlous Complications following spinal cord injury in Bangladesh; CRP, Centre for the Rehabilitation of the Paralysed; SCI, spinal cord injuries. 
CONTEXT - People with recently acquired spinal cord injuries (SCI) in Bangladesh are at high risk of developing life-threatening complications such as pressure ulcers when discharged from hospital. Pressure ulcers can be largely prevented and treated with simple ongoing strategies such as regular pressure relief, high-protein diet, urinary/bowel continence, appropriate bed overlays and wheelchair cushions combined with wound dressings and immediate bedrest at the first sign of skin breakdown. These strategies are often not adopted by people with $\mathrm{SCl}$ for a number of reasons as outlined below. In addition, people with $\mathrm{SCl}$ who are recently discharged from hospital in Bangladesh often experience ongoing problems associated with urinary and bowel incontinence, autonomic dysreflexia, pain, limited mobility, depression and loss of hope. These problems are due to limited access to medical advice, supplies and follow-up services, isolation, poor societal and family attitudes to disability, limited opportunities to return to work, limited social support and encouragement, financial strain, wheelchair inaccessible physical environments, breakdown in relationships, and limited exposure to good role models.

\begin{tabular}{|c|c|c|c|}
\hline $\begin{array}{l}\text { DESCRIPTION OF THE INTERVENTION AND ITS CAUSAL } \\
\text { ASSUMPTION } \\
\text { People with SCI do not always adopt/maintain } \\
\text { appropriate strategies and have access to appropriate } \\
\text { healthcare services important for preventing and treating } \\
\text { pressure ulcers and other complications because: } \\
\text { 1. People with SCI lack awareness and understanding of } \\
\text { what needs to be done and its importance. } \\
\text { CIVIC: The healthcare professionals use each telephone- } \\
\text { based contact and home visit to educate participants and } \\
\text { their families about what they need to do to treat and } \\
\text { prevent pressure ulcers, and other complications. } \\
\text { 2. People with SCI fail to recognise the first signs of a } \\
\text { complication and to act accordingly } \\
\text { CIVIC: The healthcare professionals use each telephone- } \\
\text { based contact and home visit to screen participants for } \\
\text { complications and to put in place an appropriate action } \\
\text { plan at the first sign of a problem. } \\
\text { 3. People with SCI experience depression, isolation and } \\
\text { loss of hope for the future which leads to complacency } \\
\text { and poor adherence } \\
\text { CIVIC: The healthcare professionals use each telephone- } \\
\text { based contact and home visit to provide regular } \\
\text { psychological and social support to participants and their } \\
\text { families, and to encourage them to get out of the home, } \\
\text { find employment, engage in purposeful activities and to } \\
\text { have hope for the future. } \\
\text { 3. People with SCI do not have sufficient funds to buy } \\
\text { appropriate wound dressings, food, urinary catheters and } \\
\text { bed mattresses. } \\
\text { CIVIC: A small amount of funding is allocated to each } \\
\text { participant. } \\
\text { 4. People with SCI do not seek assistance from existing } \\
\text { service providers, family members and the community } \\
\text { CIVIC: The healthcare professionals act as advocates for } \\
\text { the participants and help them actively seek help from } \\
\text { existing service providers, family members and the } \\
\text { community. } \\
\text { 5. Bangladesh has limited healthcare services able to } \\
\text { provide assistance for people with SCI } \\
\text { CIVIC: The healthcare professionals provide a basic } \\
\text { healthcare service. }\end{array}$ & $\begin{array}{l}\text { IMPLEMENTATION } \\
\text { Was the intervention delivered as } \\
\text { intended? Eg. Did participants receive the } \\
\text { intended number of phone calls and home } \\
\text { visits? What was the quality of the advice } \\
\text { and support provided? } \\
\text { Data sources: (1) (3) (5) (6) (7) } \\
\text { Was the control delivered as intended? } \\
\text { Eg. Was there contamination? Did the } \\
\text { control participants receive additional care } \\
\text { from CRP or elsewhere? } \\
\text { Data sources: (5) (6) (7) } \\
\text { Are the results generalisable to other } \\
\text { patients, healthcare professionals and } \\
\text { countries? How representative is the } \\
\text { sample of all patients discharged from } \\
\text { CRP? How representative is CRP of other } \\
\text { hospitals? How representative is } \\
\text { Bangladesh of other LMICs? } \\
\text { Data source: (2) (7) } \\
\text { What are the barriers and facilitators to } \\
\text { scaling up the intervention in the future? } \\
\text { Eg. What was the cost of training, service } \\
\text { delivery and infrastructure to provide the } \\
\text { service? Could regular home visits be } \\
\text { maintained? Is the intervention } \\
\text { affordable? Could the intervention be } \\
\text { incorporated into the care provided at } \\
\text { hospitals in LMICs? } \\
\text { Data sources: (7) } \\
\text { Could healthcare services employ and } \\
\text { retain staff to provide the intervention? } \\
\text { Eg. Would staff enjoy the work sufficiently } \\
\text { to prevent high staff turn over? Would } \\
\text { potential staff be sufficiently interested in } \\
\text { the work to apply for jobs? } \\
\text { Data sources: (7) }\end{array}$ & $\begin{array}{l}\text { MECHANISM of IMPACT } \\
\text { What types of issues were } \\
\text { identified during each } \\
\text { interaction? Eg. Were the } \\
\text { types of issues identified likely } \\
\text { to be amenable to advice and } \\
\text { support? Were the types of } \\
\text { issues identified likely to cause } \\
\text { complications, death and } \\
\text { depression? } \\
\text { Data sources: (1) (3) (7) } \\
\text { What was the nature of the } \\
\text { support and advice provided? } \\
\text { Eg. Was the advice and } \\
\text { support likely to be helpful? } \\
\text { Data sources: (1) (3) (4) (7) } \\
\text { What were the participants' } \\
\text { and healthcare professionals' } \\
\text { perspectives of how, why and } \\
\text { for whom the intervention } \\
\text { did or did not work? Did the } \\
\text { participants and healthcare } \\
\text { professionals believe that } \\
\text { people with SCI would benefit } \\
\text { from the intervention and if so } \\
\text { who and why? } \\
\text { Data sources: (7) } \\
\text { Would people with SCI value } \\
\text { the intervention? Eg. Would } \\
\text { people with SCI be willing to } \\
\text { continue to receive telephone- } \\
\text { based support if not part of a } \\
\text { trial? Would people with SCI } \\
\text { advocate for the intervention? } \\
\text { Data sources: (7) }\end{array}$ & $\begin{array}{l}\text { OUTCOMES } \\
\text { Outcomes Measures at } 2 \text { years } \\
\text { Primary: All-cause mortality } \\
\text { Secondary: The burden of } \\
\text { complications; Presence of } \\
\text { pressure ulcers; Severity of } \\
\text { pressure ulcers; Depression; } \\
\text { Quality of life; Independence; } \\
\text { Participation; Out-of-bed, out- } \\
\text { of-house and employment } \\
\text { Economic } \\
\text { Cost effectiveness from a } \\
\text { healthcare provider perspective } \\
\text { Maintenance/ Long term } \\
\text { impact } \\
\text { Is the CIVIC intervention } \\
\text { sustainable and can it be rolled } \\
\text { out if found to be effective? } \\
\\
\text { Data sources } \\
\text { (i) telephone interactions } \\
\text { between the healthcare } \\
\text { professionals and participants } \\
\text { (2) inclusion criteria and } \\
\text { screening logs } \\
\text { (3) chart records of each } \\
\text { interaction with intervention } \\
\text { participants } \\
\text { (4) trial records on how \$AU80 } \\
\text { per participant was spent } \\
\text { (5) data collected as part of 2- } \\
\text { year assessment indicating the } \\
\text { amount of contact with CRP } \\
\text { over the } 2 \text { years } \\
\text { (6) records held by CRP } \\
\text { indicating contact with } \\
\text { participants } \\
\text { (7) semi-structured interviews } \\
\text { with participants and healthcare } \\
\text { professionals }\end{array}$ \\
\hline
\end{tabular}

Figure 2 The process evaluation framework for the CIVIC trial. The middle blue boxes (labelled Context, Implementation and Mechanisms of Impact) include the key components of the process evaluation including exploration of the contextual factors, implementation of the trial and ways in which intervention may work. The two white boxes indicate the link between the components of the intervention and the trial outcomes. The Reach, Effectiveness, Adoption, Implementation and Maintenance and Realist frameworks guide the questions that fit within the key components of the process evaluation. The components are based on assumptions and hypotheses about how CIVIC intervention may have its effect on the primary and secondary outcomes as summarised within the two white boxes. CIVIC, Community-based InterVentions to prevent serlous Complications following spinal cord injury in Bangladesh trial; CRP, Centre for the Rehabilitation of the Paralysed; LMIC, low-income and middle-income countries; $\mathrm{SCl}$, spinal cord injury.

Our process evaluation is based on the Realist ${ }^{15}$ and Reach, Effectiveness, Adoption, Implementation and Maintenance (RE-AIM) ${ }^{16}$ frameworks. The Realist framework is a social science method of examining the relationships between the context, mechanisms and outcomes of trials involving complex interventions to better explain the possible causal pathways through which the different components of the intervention might work. It includes consideration of whom the intervention is most likely to work for and within what context along with consideration of which aspects of the intervention are most important and for what reasons. Consideration and exploration of these factors is believed to ultimately increase the uptake of research results into clinical practice.

The RE-AIM framework ${ }^{16}$ (reach, effectiveness, adoption, implementation and maintenance) uses qualitative and quantitative data to develop the intervention, and to then evaluate and disseminate trial findings. It covers five domains according to its acronym, namely: the reach of the intervention which can in part be examined by looking at those included and excluded from the trial, the effectiveness of the intervention which is reflected in the trial outcomes, the likely adoption of the intervention which can be determined by looking at how representative the site and country is of other settings, the implementation of the intervention as part of the trial which includes aspects of trial fidelity and cost containment and the maintenance of the intervention after the trial ceases. Not all aspects of the RE-AIM framework are relevant to this process evaluation but are being broadly used to guide the trial and process evaluation.

\section{Data collection and analyses}

We will use a mixed methods approach that captures both qualitative and quantitative data to address the aims of our process evaluation. All quantitative and qualitative 
Table 1 The data-collection methods that will be used to address each aim

\begin{tabular}{|c|c|c|c|c|c|c|c|}
\hline \multirow[b]{2}{*}{ Aims } & \multicolumn{7}{|c|}{ Data-collection method } \\
\hline & 1 & 2 & 3 & 4 & 5 & 6 & 7 \\
\hline \multicolumn{8}{|l|}{ Explain the trial results } \\
\hline Whether the control was delivered as intended & & & & & $\mathrm{X}$ & $\mathrm{X}$ & $\mathrm{X}$ \\
\hline $\begin{array}{l}\text { The types of issues typically identified during each interaction } \\
\text { between intervention participants and healthcare professionals }\end{array}$ & $\mathrm{x}$ & & $\mathrm{x}$ & & & & $X$ \\
\hline $\begin{array}{l}\text { Participants' and healthcare professionals' perspectives on how, why } \\
\text { and for whom the interventions did or did not work }\end{array}$ & & & & & & & $x$ \\
\hline \multicolumn{8}{|c|}{ Determine the feasibility of scaling the intervention up in Bangladesh and other low-income and middle-income countries } \\
\hline $\begin{array}{l}\text { Whether healthcare service providers could employ and retain staff to } \\
\text { provide the intervention }\end{array}$ & & & & & & & $x$ \\
\hline $\begin{array}{l}\text { Are the results generalisable to other patients, healthcare service } \\
\text { providers and countries }\end{array}$ & & $\mathrm{X}$ & & & & & $x$ \\
\hline
\end{tabular}

Legend for data-collection methods:

1. Analysis of a sample of telephone interactions between healthcare professionals responsible for providing the intervention and intervention participants.

2. Inclusion criteria and screening logs.

3. Chart audit of data collected over the trial that captures the number, length and nature of interactions between healthcare professionals and participants.

4. Audits of trial records detailing how and to whom each intervention participant's allocated \$AU80 was spent.

5. Chart audit of data collected as part of 2-year assessment indicating the amount of contact control and intervention participants had with the Centre for the Rehabilitation of the Paralysed over the 2 years.

6. Record audit of the Social Welfare Department and Community Based Rehabilitation Unit at the Centre for the Rehabilitation of the Paralysed indicating contact with control and intervention participants.

7. Semistructured interviews with participants and healthcare professionals.

data will be analysed iteratively before the final analyses of the trial results. These data will then be triangulated with the final results of the primary outcome. The type of data that will be collected to address each aim of the process evaluation is outlined in figure 2 and table 1 .

The details are as follows:

\section{Analysis of a sample of telephone interactions between} the healthcare professionals responsible for providing the intervention and the intervention participants

The purpose of these analyses will be to explore:

- Whether the intervention was delivered as intended.

- The types of issues typically identified during each interaction between the healthcare professionals and intervention participants.

- The nature of the support and advice provided by the healthcare professionals to the intervention participants.

Recordings will be taken of 20 telephone interactions between the intervention participants and the different healthcare professionals responsible for providing the intervention. Only participants who are currently in the trial at the time of data collection will be sampled. The selection of the telephone interactions will be made prior to recording and selected to ensure maximal representation of the different types of participants including: those living in rural locations versus living in urban locations; those with paraplegia versus tetraplegia; and those with minimal problems since discharge versus multiple problems since discharge. The telephone interactions will be in Bangla and will be recorded and then translated ad verbatim into English.

The recordings will be analysed using a predesigned checklist to determine how much time is spent talking directly to participants as opposed to friends or family members and how much time is spent screening for complications, providing advice, providing psychological support and engaging in social conversation. In addition, a tally will be made of the types of complications and issues that are discussed.

\section{Audit of the inclusion criteria and screening logs}

The purpose of these analyses will be to determine:

- Whether the results are generalisable to other patients, healthcare service providers and countries.

The detailed screening log kept by trial staff of all patients with SCI admitted to the Centre for the Rehabilitation of the Paralysed who are subsequently 
discharged home over the duration of the study will be examined to determine the difference between the number of patients discharged home and the number of patients ultimately randomised to the trial. These data will provide some insight into the generalisability of the results.

\section{Chart audit of data collected over the trial that captures} the number, length and nature of interactions between the healthcare professionals and participants

The purpose of these analyses will be to determine:

- Whether the intervention was delivered as intended.

- The types of issues typically identified during each interaction between intervention participants and healthcare professionals.

- The nature of the support and advice provided by the healthcare professionals to the intervention participants.

The charts kept by the healthcare professionals responsible for providing the intervention will be audited. These charts are purpose-designed forms used to record the details of every interaction with intervention participants over the course of the trial. The forms capture the type of each interaction (telephone or home visit), the length of each interaction as well as the issues discussed, key problems and advice provided during each interaction. We will use these data to determine whether intervention participants received a phone call every fortnight in the first year and every month in the second year, the median (IQR) length of each interaction and the types of complications and issues that were discussed and the type of advice provided.

Audits of trial records detailing how and to whom each intervention participants' allocated \$AU80 was spent: The purpose of these analyses will be to determine:

- The nature of the support and advice provided by the healthcare professionals.

The intervention involves the allocation of a small amount of money for each intervention participant. This money is spent according to individual needs but overseen by the healthcare professional allocated to the participant. Detailed records are kept on how this money is spent. We will audit these records to summarise the types of goods and services that are purchased and the amount of money provided to intervention participants. These data will help understand how the intervention may have its effect and the economic implications of scaling this intervention up in the future.

\section{Chart audit of data collected as part of the 2-year} assessments indicating the amount of contact control and intervention participants had with non-trial staff from the Centre for the Rehabilitation of the Paralysed since discharge The purpose of these analyses will be to determine whether:

- The control was delivered as intended.

- The intervention was delivered as intended.
At the time of protocol development, usual care was minimal. Most patients were discharged home with no formal follow-up from the Centre for the Rehabilitation of the Paralysed although sometimes patients were rung or visited on one occasion. If the results of the trial are negative then it will be important to explore whether this level of care increased since the commencement of the trial leading to contamination, and if so, whether control participants were receiving more contact from hospital staff as part of usual care than intervention participants. We will do this by tallying the number of times control and intervention participants had contact with non-trial staff from the Centre for the Rehabilitation of the Paralysed over the course of the study. These data are being collected as part of the 2-year assessments. Participants are asked how many times they have had contact with trial staff from the Centre for the Rehabilitation of the Paralysed in the preceding 2 years since discharge. We will tally these data.

\section{Record audit of the Social Welfare Department and} Community-Based Rehabilitation Unit at the Centre for the Rehabilitation of the Paralysed

The purpose of these analyses will be to determine whether:

- The control was delivered as intended.

- The intervention was delivered as intended.

We will use a second source of data to determine the amount of contact control and intervention participants had with non-trial staff from the Centre for the Rehabilitation of the Paralysed over the course of the study as part of usual care, and specifically contact with staff from the Social Welfare Department and Community Based Rehabilitation Unit at the Centre for the Rehabilitation of the Paralysed. These staff ring approximately 30 patients per month from a list of patients discharged over the last 30 years. Staff do not know which patients are part of the trial and whether those involved are control or intervention participants. The list of patients that are rung are being collected and will be used to determine how many control and intervention participants were contacted by staff not involved in CIVIC trial as part of usual care. We will also summarise any advice or follow-up care provided.

\section{Semistructured interviews with participants and healthcare} professionals

The purpose of these interviews will be to explore:

- Whether the intervention was delivered as intended.

- Whether the control was delivered as intended.

- The types of issues typically identified during each interaction between intervention participants and healthcare professionals.

- The nature of the support and advice provided by the healthcare professionals to the intervention participants.

- Participants' and healthcare professionals' perspectives on how, why and for whom the interventions did or did not work. 
- The possible barriers and facilitators to scaling the intervention up in the future.

- Whether people with SCI would value this intervention.

- Whether healthcare service providers could employ and retain staff to provide the intervention.

- Whether the results are generalisable to other patients, healthcare service providers and countries.

The interviews will be conducted by the first author (SH). He lives in Bangladesh, is fluent in Bangla and English, is a principal investigator and has worked at the Centre for the Rehabilitation of the Paralysed for over 15 years (initially as a clinical physiotherapist and then as the head of medical services). All interviews will be conducted and recorded in Bangla, and then translated ad verbatim into English (unless the healthcare professional and participant are fluent in English). The interviews will follow an interview guide which outlines broad topics to be discussed. The topics address the various purposes of the process evaluation (see online supplementary appendix 1 for types of questions that will be asked). The interviews will be conducted on a one-to-one basis and are expected to take 1-2 hours each.

Purposeful sampling will be used to select 20 participants and 14 healthcare professionals. The participants will not be the same as those used to capture the telephone interactions. Instead, they will be a mix of control and intervention participants who are either currently on the trial or have recently completed the trial, and will be selected to ensure best possible representation from a combination of the various interest groups including: living in a rural location versus living in an urban location, paraplegia versus tetraplegia, minimal problems since discharge versus multiple problems since discharge. The 14 healthcare professionals will include four staff members working on CIVIC trial who are either responsible for delivering the intervention or overall management of the trial. The remaining 10 healthcare professionals will be people not directly involved in CIVIC trial but with extensive experience or understanding of the management of people with SCI in Bangladesh. It will include people working at the Centre for the Rehabilitation of the Paralysed and working in rehabilitation in the community; and people who are in daily contact with people with SCI as well as those in managerial roles likely to have insight into the barriers and facilitators of scaling the intervention up in the future.

\section{Patient and public involvement}

People with SCI in Bangladesh were not directly involved in prioritising the research question underpinning CIVIC trial, although it is perhaps reasonable to assume that an intervention which aims to reduce mortality would be considered a priority for this group of people. As part of the process evaluation, participants in the intervention group will be asked about their experiences and perceptions of the intervention, and in particular whether they found the regular contact with healthcare professionals burdensome. We will ensure that participants of the trial are informed about the results. We will achieve this by sending them a one-page summary of the main findings in Bangla on completion of the trial.

\section{ETHICS AND DISSEMINATION \\ Ethics}

The study is being conducted in compliance with all stipulations of the study protocol, the conditions of ethics committee approval, the National Health and Medical Research Council National Statement on Ethical Conduct in Human Research (2007), ${ }^{17}$ the Note for Guidance on Good Clinical Practice (CPMP/ICH-135/95) ${ }^{18}$ and the Bangladesh Guidance on Clinical Trial Inspection (2011). ${ }^{19}$

Ethic approval will be sought for all protocol modifications. Any changes to the protocol will be updated on the registry.

\section{Dissemination}

CIVIC trial will provide unbiased and precise estimates of the effectiveness and cost-effectiveness of an inexpensive and sustainable model of community-based care for people with SCI in Bangladesh. Evidence of effectiveness and cost-effectiveness will have widespread implications for provision of health services for people with SCI and other conditions that cause serious disability in low-income and middle-income countries.

Process evaluations conducted alongside trials involving complex interventions such as that provided in CIVIC are widely advocated because the causal links between the different components of the intervention and outcomes of the trial are not always clear. The intervention provided as part of CIVIC is a complex community-based rehabilitation intervention that is based on similar services provided in high-income countries and studies which have advocated the benefits of telephone-based support for people with SCI. ${ }^{20}{ }^{21}$ The intervention is complex because it involves repeated interactions between healthcare professionals and intervention participants over a 2-year period as well as the provision of a small amount of financial support. Details about the intervention have been described in our trial protocol according to the Template for Intervention Description and Replication checklist. ${ }^{22}$ Importantly, attention has been directed at ensuring the intervention is delivered as intended. For this reason, trial staff are regularly trained and provided with standard forms and screening logs which are completed each time they have contact with an intervention participant. However, it is not possible nor is it desirable to ensure that all interactions between trial staff and intervention participants are exactly the same. The trial is pragmatic and hence trial staff are expected to individualise their interactions with intervention participants according to their many diverse needs. The nature of each interaction will also depend on the personalities of both the trial staff and the intervention participants. Some interactions may be largely social and the trial staff may not obviously provide 
any advice or support. However, regular contact with a concerned and supportive healthcare professional may be important for people who otherwise have very little contact with healthcare professionals and may be socially isolated. The skills of staff may also differ in keeping with the pragmatic nature of the trial and reflecting the realities of how this intervention would be provided in the future, if successful. Some staff may be very skilled at providing advice and support while others may not be as skilled. It is important to explore the nature of the interactions to better understand how the skill of staff may or may not influence outcomes and how the intervention may or may not work. As such, our process evaluation will look at some of these key contextual factors that both contribute to and hinder the potential benefit of the intervention and that are important for understanding different aspects of the intervention.

The intervention includes the allocation of a small amount of money for each participant to spend on services and goods such as dressings for pressure ulcers, catheters for bladder management, mattresses for beds and transport for medical attention. It will be important to determine how this money was spent as part of the process evaluation and whether it was considered an essential and important aspect of the intervention. These data will also provide insight into the economic implications of living with SCI in a country like Bangladesh and the role poverty plays in complications, mortality, depression and quality of life. Answers to these questions will help us better understand whether financial assistance is an essential aspect of the intervention and needs to be included when scaling up the intervention.

An important barrier to scaling the intervention up in the future if it is found to be effective will be cost. While a formal economic analysis from the healthcare provider perspective will be performed as part of the trial, the results of this process evaluation will also provide insight into economic barriers to scaling up of the intervention. So, interviews with healthcare professionals involved in management will explore their perspectives on the financial constraints and implications of rolling out the intervention. We will combine this information with the results of the formal economic analysis to make recommendations on the overall financial implications of scaling up the intervention across Bangladesh and other low-income and middle-income countries. We have done similar for a trial designed to determine the effectiveness of family-led rehabilitation following stroke in India (ATTEND trial). ${ }^{23}$ The process evaluation that formed part of the ATTEND trial has guided the process evaluation for CIVIC trial.

In all, our process evaluation will be an important aspect of CIVIC trial. It will explore facilitators and barriers to rolling this intervention out in the future if it is found to be effective. Regardless of CIVIC trial results, our process evaluation will help guide future research in this much-neglected area.
Author affiliations

${ }^{1} J o h n$ Walsh Centre for Rehabilitation Research, Kolling Institute, Sydney Medical School/Northern, University of Sydney, Sydney, New South Wales, Australia

${ }^{2}$ George Institute for Global Health, Sydney, New South Wales, Australia

${ }^{3}$ Centre for the Rehabilitation of the Paralysed, Savar, Bangladesh

${ }^{4}$ Livability International, Fermanagh, Northern Ireland

${ }^{5}$ Clinic for Spinal Cord Injuries, University of Copenhagen, Hornbæk, Denmark

${ }^{6}$ Indian Spinal Injuries Centre, New Delhi, India

Contributors MSH, LAH, IDC, RLL, SJ and SM were responsible for the design of the intervention and the trial. MSH, LAH, IDC, RLL, SJ, SM FB-S and HSC secured funding. SJ was responsible for the economic analyses. SJ, HL, MSH and LAH were responsible for the design of the process evaluation. MSH and HL were responsible for collection of the qualitative data. MSI, SM and MAR were responsible for the local site. All authors have read and approved the final manuscript.

Funding The trial is funded by a 5 -year grant from the National Health and Medical Research Council of Australia (APP1080259).

Competing interests None declared.

Patient consent Not required.

Ethics approval The human ethics review committees of the Centre for the Rehabilitation of the Paralysed, Bangladesh (CRP-R\&E-0401-126), and The University of Sydney, Australia (2015/041).

Provenance and peer review Not commissioned; peer reviewed for ethical and funding approval prior to submission.

Open access This is an open access article distributed in accordance with the Creative Commons Attribution Non Commercial (CC BY-NC 4.0) license, which permits others to distribute, remix, adapt, build upon this work non-commercially, and license their derivative works on different terms, provided the original work is properly cited, appropriate credit is given, any changes made indicated, and the use is non-commercial. See: http://creativecommons.org/licenses/by-nc/4.0/.

\section{REFERENCES}

1. World Health Organization. International Perspectives on Spinal Cord Injury. Geneva: World Health Organization, 2013.

2. Elshahidi MH, Monir NY, Elzhery MA, et al. Epidemiological characteristics of traumatic spinal cord injury (TSCl) in the MiddleEast and North-Africa (MENA) Region: A systematic review and meta-analysis. Bull Emerg Trauma 2018;6:75-89.

3. Wyndaele M, Wyndaele JJ. Incidence, prevalence and epidemiology of spinal cord injury: what learns a worldwide literature survey? Spinal Cord 2006;44:523-9.

4. Chamberlain JD, Meier S, Mader L, et al. Mortality and longevity after a spinal cord injury: systematic review and meta-analysis. Neuroepidemiology 2015;44:182-98.

5. Øderud T. Surviving spinal cord injury in low income countries. Afr J Disabil 2014;3:80.

6. Barman A, Shanmugasundaram D, Bhide R, et al. Survival in persons with traumatic spinal cord injury receiving structured follow-up in South India. Arch Phys Med Rehabil 2014;95:642-8.

7. Gosselin RA, Coppotelli C. A follow-up study of patients with spinal cord injury in Sierra Leone. Int Orthop 2005;29:330-2.

8. Hossain MS, Rahman MA, Herbert RD, et al. Two-year survival following discharge from hospital after spinal cord injury in Bangladesh. Spinal Cord 2016;54:132-6.

9. Hossain MS, Rahman MA, Bowden JL, et al. Psychological and socioeconomic status, complications and quality of life in people with spinal cord injuries after discharge from hospital in Bangladesh: a cohort study. Spinal Cord 2016;54:483-9.

10. Zakrasek EC, Creasey G, Crew JD. Pressure ulcers in people with spinal cord injury in developing nations. Spinal Cord 2015;53:7-13.

11. Levy LF, Makarawo S, Madzivire D, et al. Problems, struggles and some success with spinal cord injury in Zimbabwe. Spinal Cord 1998;36:213-8.

12. Hossain MS, Harvey LA, Rahman MA, et al. A pilot randomised trial of community-based care following discharge from hospital with a recent spinal cord injury in Bangladesh. Clin Rehabil 2017;31:781-9.

13. Hossain MS, Harvey LA, Rahman MA, et al. Community-based InterVentions to prevent serlous Complications (CIVIC) following spinal cord injury in Bangladesh: protocol of a randomised controlled trial. BMJ Open 2016;6:e010350. 
14. Moore GF, Audrey S, Barker M, et al. Process evaluation of complex interventions: medical research council guidance. BMJ 2015;350:h1258.

15. Magnin $\mathrm{M}$, Jeannot $\mathrm{E}$, Voahangy $\mathrm{R}$, et al. A realistic evaluation approach highlighted the success factors and difficulties of an innovative and comprehensive malnutrition programme in Madagascar. Acta Paediatr 2018.

16. Gaglio B, Phillips SM, Heurtin-Roberts S, et al. How pragmatic is it? Lessons learned using PRECIS and RE-AIM for determining pragmatic characteristics of research. Implement Sci 2014;9:96.

17. NHMRC. National Statement on Ethical Conduct in Research Involving Humans. Secondary National Statement on Ethical Conduct in Research Involving Humans. 2007 http://www.nhmrc.gov. au/guidelines/publications/e72

18. The Therapeutic Goods Administration. CPMP/ICH Note for guidance on good clinical practice (CPMP/ICH-135/95). Canberra, 2000.
19. Directorate General of Drug Administration Clinical Trial Inspection. Guidance on clinical trial inspection. Bangladesh, 2011.

20. Hoffman JM, Burns S, Bombardier CH. Scheduled telephone followup to improve outcomes after spinal cord injury rehabilitation. Top Spinal Cord Inj Rehabil 2013;19:26.

21. Dorstyn D, Mathias J, Denson L. Applications of telecounselling in spinal cord injury rehabilitation: a systematic review with effect sizes. Clin Rehabil 2013;27:1072-83.

22. Hoffmann TC, Glasziou PP, Boutron I, et al. Better reporting of interventions: template for intervention description and replication (TIDieR) checklist and guide. BMJ 2014;348:g1687.

23. Lindley RI, Anderson CS, Billot L, et al. Family-led rehabilitation after stroke in India (ATTEND): a randomised controlled trial. Lancet 2017;390:588-99.

24. Liu H, Lindley R, Alim M, et al. Protocol for process evaluation of a randomised controlled trial of family-led rehabilitation post stroke (ATTEND) in India. BMJ Open 2016;6:e012027. 